University of San Diego

Digital USD

Spring 5-23-2017

\title{
The ABCs of ABA: Comparing Consequence Preferences for Maladaptive Behavior in Children on the Autism Spectrum
}

Alyssa A. Serceki

University of San Diego

Follow this and additional works at: https://digital.sandiego.edu/honors_theses

Part of the Social and Behavioral Sciences Commons

\section{Digital USD Citation}

Serceki, Alyssa A., "The ABCs of ABA: Comparing Consequence Preferences for Maladaptive Behavior in Children on the Autism Spectrum" (2017). Undergraduate Honors Theses. 38.

https://digital.sandiego.edu/honors_theses/38

This Undergraduate Honors Thesis is brought to you for free and open access by the Theses and Dissertations at Digital USD. It has been accepted for inclusion in Undergraduate Honors Theses by an authorized administrator of Digital USD. For more information, please contact digital@sandiego.edu. 
The ABCs of ABA:

Comparing Consequence Preferences for Maladaptive Behavior in Children on the Autism Spectrum

\begin{abstract}
A Thesis
Presented to

The Faculty and the Honors Program

Of the University of San Diego

By

Alyssa Ann Serceki

Department of Psychological Sciences
\end{abstract}




\begin{abstract}
Applied Behavior Analysis, or ABA, is a common therapeutic technique that has been implemented since the 1960 s as a method of teaching appropriate social and functional behaviors to children on the Autism Spectrum and related disorders. ABA focuses on altering behavior by controlling the Antecedents and Consequences related to the targeted Behavior. ABA is only implemented by trained practitioners, who select from a variety of Consequence options when determining how to modify a given child's Behavior. Because ABA is rooted in Behavioral Psychology and techniques of learning theory, it is possible that Psychological education may play a role in the determination of appropriate consequences for the child. The aim of the present study was to determine whether differential exposure to Psychological concepts (operationalized by the number of Psychology courses taken) would impact preference for certain Consequences. Participants were asked to read 3 situations describing a child's behavior and rank their preference of the Consequence to be administered from a set of provided options. Consistent with hypotheses, students with more exposure to Psychological concepts (e.g., $5+$ courses or a Cognitive course) displayed different Consequence preferences from those not exposed and were more likely to select an appropriate Consequence. Unexpectedly, this result appeared primarily in Situation 3. The results suggest that further exploration is warranted and that a Psychological education can influence ABA consequence choices.
\end{abstract}




\section{The ABCs of ABA:}

\section{Comparing Consequence Preferences for Maladaptive Behavior in Children on the Autism Spectrum}

As a Behavior Interventionist (B. I.), or a social and behavioral therapist for children on the Autism Spectrum and related disorders, I am trained to work with children using Applied Behavior Analysis (ABA) therapy. ABA is the use of specific techniques designed to create a positive change in the behavior of those on the Autism Spectrum and related disorders, using techniques such as positive reinforcement. For example, when I am working with clients, I often use what I call my "B. I. Voice,” or the perky, upbeat voice I use to deliver praise, one form of positive reinforcement. This praise, delivered as a consequence for engaging in desired behavior, helps increase the occurrence of the behavior. Because consequences play a large role in maintaining behavior, $\mathrm{ABA}$ utilizes many consequences beyond just positive reinforcement to alter behavior and the choice of consequence is often determined as a combination of provider preference and child characteristics. One provider characteristic is educational background. The current study sought to explore how differential exposure to one aspect of education, collegiate Psychology courses, might impact the preferred Consequences chosen by students to decrease the occurrence of future maladaptive behavior.

\section{The Basics of Applied Behavior Analysis}

ABA is a type of intervention that focuses on teaching appropriate social behaviors to individuals on the Autism Spectrum and related disorders, gained popularity during the 1960s. As the term implies, ABA utilizes the principles of behaviorism to 
increase desirable and decrease undesirable behaviors in order to promote more effective functioning (“Applied Behavior Analysis”).

In order to decrease the maladaptive behaviors, an ABA practitioner would observe the behavior occurring during a natural environment and focus on analyzing not just the behavior, but what precedes and follows it. This is referred to as functional analysis, or analyzing the $\mathrm{ABCs}$. The functional analysis breaks down the behavior into the Antecedent, Behavior, and Consequence ("ABC's of Applied Behavior Analysis"). The Antecedent is what occurs directly before a behavior, for example, a request or command such as requiring a child to clean up their toys, or a feeling, like if the child is hungry. The Behavior is what occurs as a result of the Antecedent, and in this context, would be considered maladaptive. For example, a behavior could be defined as selfinjurious behavior like biting or banging head against the floor or the wall, or could relate to tantrums like excessive screaming or crying. The Consequence is what occurs as a result of the behavior, and could presumably be modified to help decrease the occurrence of this maladaptive behavior. Interventions can be done on the Antecedent as well, but the focus of this study is on consequence-based interventions only.

\section{Four Main Reinforcers for Behavior}

In traditional operant conditioning (which underlies the logic of ABA interventions), consequences can be considered either punishing or reinforcing. A consequence is considered punishment when the behavior that evokes it decreases in response to the consequence while a consequence is considered reinforcing when the behavior that evoked it increases or persists. ABA identifies four main reinforcers for maladaptive behavior. These four different types of reinforcers are used to determine the 
cause of the behavior, as well as why the behavior persists. These types of reinforcers are classified into two different categories, Social and Automatic Reinforcement. Social Reinforcement is related to reinforcement from interactions with others, and Automatic Reinforcement is related to reinforcement from interactions with one's self.

Additional classification is based on what occurs during the consequence and can be considered Positive or Negative. Positive reinforcement refers to the addition of something whereas Negative reinforcement refers to the removal of something from the situation. For example, if the cause of the behavior is determined to be due to Positive Social Reinforcement, this means that the behavior occurs due to the desire to have attention or access to something that is desired, requiring social interaction with another. Whereas if the cause of the behavior is determined to be due to Negative Social Reinforcement, this means that the behavior is due to the desire to escape something that is undesired, requiring the removal of social interaction with another. Specifically, if a child screams at their parent because they want a cookie, and then they receive that cookie and stop screaming, the behavior is reinforced due to Positive Social Reinforcement because they are being granted access to something they want as a result of their behavior. Alternatively, if a child is asked to clean up their toys and they don't want to, they might run away to escape cleaning up (Negative Social Reinforcement), which reinforces their behavior and increases the likelihood that they will try to escape other undesired tasks in the future. These are considered Social Reinforcements because they require someone else to be reinforcing, and are done in order to attain an object or attention, or to escape something undesired. 
In much the same pattern, if the cause of the behavior is determined to be due to Positive Automatic Reinforcement, this means that the occurrence of the behavior itself is reinforcing. For example, many children on the Spectrum find hand flapping to be incredibly reinforcing; they get enjoyment from just flapping their hands. If the behavior is determined to be due to Negative Automatic Reinforcement, this means that the occurrence of the behavior is due to pain attenuation. For example, if a child is hitting their mouth repeatedly, it could be a manner of distracting themselves from a toothache or other pain. These are considered Automatic Reinforcements because they don't require anyone else to be reinforcing, but are done by the individual to him or herself in order to attain enjoyment or escape pain. The cause of the child's maladaptive behavior is imperative to determine when trying to decide the proper consequence in order to decrease the occurrence of such behavior.

\section{Alternative Intervention Strategies}

Evidence has shown that early therapeutic treatment is the most effective in improving behavior outcomes. Although timely access is incredibly important, it can be difficult for clients to receive the desired treatment due to long waiting lists, or even lack of resources, as many of these therapies are expensive and time-consuming. As a result, many therapists are seeking different methods of teaching parents the necessary skills to conduct therapy at home, in order to gain the necessary improvements as early as possible. Two such therapies are Parent-Child Interaction Therapy and Pivotal Response Training.

PRT, or Pivotal Response Training focuses on increasing communication in children using natural, daily-life settings such as play time (Coolican, Smith \& Bryson, 
2010). PRT utilizes many principles of ABA, "which assume that children's impairments can be improved with environmental manipulations, such as reinforcement, consequences, and extinction" (Baker-Ericzén, Stahmer \& Burns, 2007). While based on ABA principles, PRT focuses on "pivotal areas," such as self-initiation, which are important areas in a range of behaviors, and when these areas are targeted, they can cause improvement in many different behaviors related to these pivotal areas. Evidence demonstrates that PRT can be successful in increasing language, social, and play skills in children with autism, as well as decreasing undesired behaviors by focusing on naturallyoccurring opportunities and consequences (Baker-Ericzén et al., 2007).

PCIT, or Parent-Child Interaction Therapy is another type of therapy that attempts to decrease the occurrence of maladaptive behavior in children, particularly those with behavioral disorders. "Externalizing behaviors are a common component of the clinical presentation of autism spectrum disorders. Although traditionally used with typicallydeveloping children, parent-child interaction therapy is one behaviorally-based parent training program that has demonstrated success in increasing child compliance, reducing problem behavior, and improving parent-child communication" (Masse, McNeil, Wagner \& Quetsch, 2016). Through PCIT, parents are taught how to utilize selective attention and positive reinforcement in order to shape their child's behavior. For example, parents are taught to provide selective attention, like ignoring inappropriate behavior and delivering praise (positive reinforcement) contingent on the child's compliance or displays of appropriate behavior. Parents are also taught implementation of effective commands in order to increase compliance (Masse et al., 2016). Both PCIT and Pivotal Response Training focus on consistently using these skills in a naturalistic setting, using 
natural consequences and reinforcement, in order to increase compliance and communication while decreasing problem behavior in children. However, for the purposes of this study, the focus is mainly on Applied Behavior Analysis and the importance of consequences of reinforcement on maladaptive behavior.

\section{The Current Study}

Because there are many ways to alter the consequence related to a behavior, determining the factors that might influence the choice of which consequence gets implemented is an important area of study. To my knowledge, no previous research has attempted to investigate the preferences in choice of consequence for maladaptive behaviors in children on the Autism Spectrum and related disorders. Thus, the aim of the current study was to explore whether educational background might affect the preference of Consequences. Because the principles of ABA are based in Psychology- specifically learning theory- it was of interest to determine whether differential exposure might influence the consequences that were chosen to alter behavior.

\section{Hypothesis}

Based on this logic, it was hypothesized that the students who had taken or were taking at least 5 classes or at least one Cognitive Psychology class would demonstrate preferences that were different from those not so classified. Although not necessarily explicitly exposed to ABA directly, Psychology students who are far along in their education or have taken a Cognitive Psychology class have been previously exposed to reinforcement concepts at length, and therefore might have a better understanding of what would be appropriate for altering undesirable behavior. The second hypothesis was that the Consequence options that were inappropriate based on $\mathrm{ABA}$ techniques would be 
preferred the least and that Psychological education would increase the likelihood of selecting an appropriate Consequence option compared to not having been exposed to Psychological concepts.

\section{Method}

\section{Participants}

Participants were students enrolled in various Psychology courses, both upper division and lower division, at the University of San Diego. Participation in this study was voluntary and participants were recruited by contacting course instructors, who made announcements about the study in the classroom. The study initially consisted of 85 participants, both male and female (17 males and 68 females), but 14 students were dropped from analysis for failing to complete the entire survey, leaving 71 participants (16 males and 55 females) between the ages of 18-28. Of these 71 participants, 37 were Freshmen, 12 were Sophomores, 9 were Juniors, 10 were Seniors, and 3 responded with "Other." The participants were further classified into 49 Lowerclassmen and 22 Upperclassmen (the 3 participants that indicated "Other" were classified by the Researcher as Upperclassmen given the amount of coursework they indicated as having

completed). 25 participants indicated that they were Majors or Minors in Psychology and 46 indicated that they were not (selecting either "No" or "I don't know" were both classified as "No" for the purposes of this study). Eleven participants indicated that they had taken, or were currently taking a Cognitive course while 60 indicated that they had not. Eighteen participants indicated that they had taken or were currently taking more than 5 psychology classes, while 53 indicated that they had taken less than 5. 


\section{Materials}

A survey was created by the author consisting of 10 questions, including demographic information as well as questions pertaining to the participants' Consequence preferences.

Demographic items. Seven questions pertained to demographic information about the participants. They were asked to report their gender, age, class standing, if they were a parent, how many children they had, whether they were majoring or minoring in Psychology, as well as how many Psychology classes they had previously taken or were currently taking. Response options were either fixed alternative (e.g., gender: male, female, other, prefer not to say) or open-ended.

Scenarios. The final three items on the questionnaire were designed to assess the participants' preferences for consequences. In these items, a situation was described providing both the Antecedent and Behavior for an imaginary child on the Autism Spectrum and a list of 5 possible Consequences. Two of the Consequences provided would not be administered in ABA while three of the options provided could be. The participants were asked to rank the preference among the 5 Consequences, from 1 being the most preferred option and 5 being the least preferred option. (For full scenarios and Consequences, please see Appendix A).

\section{Procedure}

As a cover story, participants were told that the purpose of the study was to determine how college students and parents would respond to an incident involving consequences for children on the Autism Spectrum using Applied Behavior Analysis 
(ABA) tactics. Participants were told that they were being asked to take part in this study because the author was interested in comparing student preferences to those of parents. These instructions were included in order to shift the focus away from psychological education as a primary variable of study. Participants were informed that participation was completely voluntary and that if they chose to participate, they were free to withdraw at any time without penalty.

Upon accessing the survey via Qualtrics, participants were first presented with the informed consent, then asked to complete demographic information, followed by instructions describing that they would read scenarios and be asked to rank the consequences in order of preference. After moving through the three scenarios, the final screen provided a short debrief to dispel the notion that parental responses were being recorded and indicated that differential exposure to psychology education was the variable under investigation. The survey took approximately 6 minutes to complete.

\section{Results}

As indicated above, two of the five response options provided were inappropriate according to ABA principles. Thus, participants' responses were analyzed two ways. First, the top choice for each participant based on gender, class standing, and psychological exposure was evaluated using Chi Square analysis. Secondly, to more directly evaluate the hypotheses, the participants' responses were coded as either correct or incorrect based on whether an appropriate or inappropriate option was selected as their top choice. This data was then also subjected to Chi Square analysis to determine whether there were different preferences based on gender, class standing, and psychological exposure. 


\section{Gender}

To determine whether there was a difference in choice of Consequence based on gender, participants' top choices were analyzed using a Chi Square test of independence for each situation. For Situation 1, the results indicated no significant difference for males compared to females, $\chi^{2}(4)=0.35, p>0.05$. Similarly, for Situation 2 and Situation 3, males and females did not report different preferences, $\chi^{2}(4)=6.20, p>0.05$ and $\chi^{2}(4)=7.27, p>0.05$ respectively.

In order to determine whether there was a difference between appropriate Consequence preferences based on gender, the recorded responses (appropriate v. inappropriate) were analyzed using a Chi Square test of independence for each situation. For Situation 1 and 3, the results indicated no significant difference in selection of appropriate v. inappropriate Consequence based on gender, $\chi^{2}(1)=0.15, p>0.05$ and $\chi^{2}(1)=0.387, p>0.05$, respectively. However, for Situation 2 , the results indicated a significant difference in appropriate preference, $\chi^{2}(1)=4.67, p<0.05$. Males were more likely to select an appropriate option.

\section{Class Standing}

To determine whether there was a difference in choice of Consequence based on Upperclassmen and Lowerclassmen class standing, the top choice preferences were analyzed using a Chi Square test of independence for each situation. For Situation 1 and Situation 2, the results indicated no significant difference for Upperclassmen compared to Lowerclassmen, $\chi^{2}(4)=7.29, p>0.05$ and $\chi^{2}(4)=1.84, p>0.05$, respectively. However, for Situation 3, the results indicated a significant difference in preferences, $\chi^{2}(4)=12.63$, 
$\mathrm{p}<0.05$. The highest proportion of Lowerclassmen selected Consequence $3(42.9 \%)$ whereas the highest proportion of Upperclassmen selected Consequence 2 (45\%).

In order to determine whether there was a difference between appropriate Consequence preferences based on Upperclassmen and Lowerclassmen class standing, the recorded responses (appropriate v. inappropriate) were analyzed using a Chi Square test of independence for each situation. For Situation 1 and Situation 3, the results indicated a significant difference in selection of appropriate Consequence based on class standing, $\chi^{2}(1)=5.10, p<0.05$ and $\chi^{2}(1)=5.31, p<0.05$, respectively. Upperclassmen were more likely to select an appropriate option. For Situation 2, the results indicated no significant difference in selection of appropriate v. inappropriate Consequence based on class standing, $\chi^{2}(1)=0.074$, and $\mathrm{p}>0.05$.

\section{Psychology Major/Minor Status}

A Chi Square test of independence was used to determine if there was a difference in choice of Consequence based on whether or not the student was a Psychology Major or Minor for each situation. For Situation 1, Situation 2, and Situation 3, the results indicated no significant difference in preference indicated by those who were Psychology Major or Minor and those who were not, $\chi^{2}(4)=4.96, p>0.05, \chi^{2}(4)=3.90, p>0.05$, and $\chi^{2}(4)=9.15, p>0.05$, respectively.

A Chi Square test of independence was used to determine if there was a difference in appropriate Consequence preferences between the students who indicated that they were Psychology Major or Minors and the students who did not indicate such status based on the recorded responses (appropriate v. inappropriate). For Situation 1, the results showed $\chi^{2}(1)=4.54, p<0.05$, meaning there was a significant difference between 
the appropriate preferences indicated by those who were Major/Minors and those who were not for Situation 1. Analysis of the data indicated that, as expected, more Majors/Minors (72.0\%) selected an appropriate choice compared to non-Majors/Minors (45.7\%). Contrary to hypothesis, for Situation 2 and Situation 3, the results indicated no significant difference in appropriate Consequence preference, $\chi^{2}(1)=0.88, p>0.05$ and $\chi^{2}(1)=2.92, p>0.05$. For display of percentages, see Figure 1.

\section{Psychological Exposure}

Number of psychology courses taken. Given one of the primary hypotheses was that differential exposure to psychological concepts would impact preferences, students were grouped based on the number of Psychology courses they had reported having taken. Specifically, students were divided into two groups, those who had taken less than 5 Psychology courses and those who had taken 5 or more.

In order to determine whether there was a difference in Consequence preferences based on whether the participant had taken at least 5 Psychology classes, the top choice preferences were analyzed using a Chi Square test of independence for each situation. For Situation 1 and Situation 3, the results indicated a significant difference for the students who had taken at least 5 Psychology courses and those who had not, $\chi^{2}(4)=$ $12.86, p<0.05$ and $\chi^{2}(4)=10.96$, and $p<0.05$, respectively. For Situation 1 , the highest proportion of students who had not taken at least 5 Psychology classes selected Consequence 2 and Consequence 3 (28.3\% and $37.7 \%$ respectively) and the highest proportion of students who had taken at least 5 Psychology classes selected Consequence $4(33.3 \%)$, though the preferences were more evenly distributed. For Situation 3, the highest proportion of students who had not taken at least 5 Psychology classes selected 
Consequence 3 (41.5\%), and the highest proportion of students who had taken at least 5 Psychology classes selected Consequence 2 (35.5\%). However, for Situation 2, the results indicated no significant difference in preferences between students who had taken at least 5 Psychology courses and those who had not, $\chi^{2}(4)=2.17, p>0.05$.

In order to determine whether there was a difference in appropriate Consequence preferences between those Psychology students who had taken at least 5 classes and those who had not, the recorded responses (appropriate v. inappropriate) were analyzed using a Chi Square test of independence for each situation. Contrary to hypothesis, for Situation 1 and Situation 2, the results indicated no significant difference in selection of appropriate Consequence preference based on taking at least 5 Psychology classes, $\chi^{2}(1)=$ 2.91, $p>0.05$ and $\chi^{2}(1)=1.78, p>0.05$, respectively. However, for Situation 3, the results indicated a significant difference in appropriate preference, $\chi^{2}(1)=6.30, p<0.05$. As predicted, those who had taken at least 5 Psychology courses were more likely to select an appropriate option (70.6\%) compared to those who had not (35.8\%). For display of percentages, see Figure 2.

Cognitive Psychology. Finally, it was predicted that having taken a Cognitive Psychology course, which exposes participants to learning principles, would impact preferences. Students were classified as either having taken a Cognitive Psychology course or not and their top preference for each scenario were then analyzed using a Chi Square test of independence. For Situation 1 and Situation 3, the results indicated a significant difference for those who had taken a Cognitive course and those who had not, $\chi^{2}(4)=24.54, p<0.05$ and $\chi^{2}(4)=13.45, p<0.05$, respectively. For Situation 1 , the highest proportion of students who had not taken a Cognitive course selected 
Consequence 2 and Consequence $3(30.0 \%$ and $35.0 \%$ respectively) and the highest proportion of students who had taken a Cognitive course selected Consequence 4 (54.5\%). For Situation 3, the highest proportion of students who had not taken a Cognitive course selected Consequence 3 (40.7\%), and the highest proportion of students who had taken a Cognitive course selected Consequence 2 and Consequence 5 (both were 27.3\%), though the preferences were very equally distributed. However, for Situation 2, the results indicated no significant difference for those who had taken a Cognitive course and those who had not, $\chi^{2}(4)=5.70, p>0.05$.

In order to determine whether taking a Cognitive course had an effect on appropriate Consequence preference, the recorded responses (appropriate v. inappropriate) were analyzed using a Chi Square test of independence for each situation. For Situation 1 and Situation 2, the results indicated no significant difference for those who had taken a Cognitive course and those who had not, $\chi^{2}(1)=1.67, p>0.05$ and $\chi^{2}(1)=1.66, p>0.05$, respectively. However, for Situation 3, the results indicated a significant difference in appropriate Consequence preference for those who had taken a Cognitive course and those who had not, $\chi^{2}(1)=4.30, p<0.05$. Consistent with prediction, those who had taken a Cognitive course preferred an appropriate consequence (72.7\%) to the inappropriate ones, whereas those who had not taken such a course showed the opposite pattern (39.0\%). For display of percentages, see Figure 3.

\section{Discussion}

This study intended to determine whether differential exposure to Psychological concepts (operationalized by the number of Psychology courses taken) would impact preference for certain Consequences to control unwanted behavior. It was hypothesized 
that there would be a difference between students who had taken at least 5 Psychology classes and those who had taken less than 5 . The results of this study demonstrated that there was a difference in preference between these two groups of students, in that students who had taken at least 5 Psychology classes selected different top Consequence preferences in Situations 1 and 3 compared to students who had not taken 5 Psychology classes. Additionally, it was hypothesized that the two options which were not ABAappropriate would be selected the least and more so by those with increased Psychological exposure. The results were consistent with this prediction, but only for Situation 3.

Additionally the hypotheses related to Cognitive Psychology exposure were partially supported. The results demonstrated that there was a difference in preference between those who had and had not taken a Cognitive Psychology course in that students who had taken at least one Cognitive course selected different top Consequences in Situations 1 and 3 than those who had not taken a Cognitive course. Similarly, students who had taken at least one Cognitive course were more likely to select an appropriate Consequence option for Situation 3.

The current project is one of the first studies to evaluate Consequence preferences among lay individuals. The main aim was to explore whether exposure to Psychological concepts would impact the preference chosen by participants. The evidence suggests that having exposure to a Psychological education does influence the perceived appropriateness of certain consequences. While the findings were far from consistent, several results indicated that students who had taken multiple Psychology classes, or had taken a Cognitive course, were more likely to select an appropriate response. 
Students were also more likely to select an appropriate response for Situation 3 than for Situations 1 and 2. This could be due to the exposure to the concepts, as each Situation was presented to participants in the same order. Thus, it is possible that by the third time students were exposed to the information, they might have been more comfortable with the concept of appropriate Consequences. Situation 3 also incorporated an element that is common in ABA therapy, the use of a physical schedule, which might have helped students understand the situation from an $\mathrm{ABA}$ perspective, thus increasing the chance of choosing an ABA-appropriate Consequence.

\section{Potential Limitations}

This study does have some limitations, the most obvious being sample size. The survey was only administered to Psychology students at the University of San Diego. Due to convenience, only certain professors the researcher knew well were asked to disseminate the link to their students for completion. Thus, the results may be less generalizable to the larger pool of all Psychology students. Future research could address this issue by disseminating the survey more widely.

The survey itself was completed online via Qualtrics and was completed by participants in various locations. Thus, it is impossible to know how seriously participants approached the questions and whether or not they were distracted by other things while completing it. Additionally, students taking the survey from the same class could easily have discussed it with others and thus some participants may have had prior knowledge of the questions and what to expect.

Finally, students were much more likely to respond with an appropriate preference for Situation 3. Although it is unclear as the reason why, it is possible that 
factors included in the scenario cued participants to more appropriate options. The potential difference between Situation 3 and the other two does not invalidate the findings however, as the differences found were all in the predicted direction. Future research should more carefully control the information provided in each scenario to address this issue.

\section{Future Directions and Implications}

Because this study was exploratory, there is much opportunity for further exploration. Future studies designed to further explore additional variables that might impact the selection of Consequence preferences could help researchers more fully understand what underlies Consequence choices. Because some Consequences are likely more effective than others, evaluating whether and how provider variables might impact Consequence choice has therapeutic implications. Additionally, since the Consequence preferences for Scenario 3 were often more appropriate than for Situation 1 and 2, future studies could explore whether a practice/exposure effect operates in Consequence selection. Perhaps exposure to Psychological concepts, not just through education, but by becoming more familiarized with the concepts themselves over the course of being tested using multiple Situations, has an effect on selection of appropriate Consequence preferences.

Future studies should also attempt to incorporate a larger sample as highlighted above. One possibility would be to include a wider range of psychological exposure to more fully determine whether there is some minimum amount of classes necessary to influence Consequence preferences in the appropriate direction. 
Finally, although the parent-student comparison was created as a cover story, since parents are often involved in the implementation of treatment, future work exploring parent variables that influence Consequence preferences may also be useful. For example, would a small exposure to Psychological education influence parents to select more appropriate choices, or does length of time interacting a particular child impact such a choice?

\section{Conclusion}

Care should be taken when drawing strong conclusions from this research given its exploratory nature. However, the relationship found between exposure and preference suggests that Psychological education may be an important determinant of ABA consequence selection. Both differential exposure to Psychology courses (5 or more) and a Cognitive course altered Consequence selection among participants. Future studies designed to more fully explore this topic, as outlined above, could prove useful on both a theoretical and practical level. Perhaps, unsurprisingly, given its grounding in Behavioral Psychology, ABA implementation appears to be influenced by Psychological exposure. 


\section{References}

ABC's of ABA, ABC's of Applied Behavior Analysis. (n.d.). Retrieved September 30, 2016, from https://www.abcsofaba.com/aba

Applied Behavior Analysis (ABA). (n.d.). Retrieved September 20, 2016, from https://www.autismspeaks.org/what-autism/treatment/applied-behavior-analysisaba

Applied Behavioral Strategies - Reinforcement 101. (n.d.). Retrieved September 30, 2016, from http://www.appliedbehavioralstrategies.com/reinforcement-101.html

Baker-Ericzén, M. J., Stahmer, A. C., \& Burns, A. (2007). Child demographics associated with outcomes in a community-based Pivotal Response Training program. Journal Of Positive Behavior Interventions, 9(1), 52-60.

Bjørseth, A., \& Wichstrøm, L. (2016). Effectiveness of Parent-Child Interaction Therapy (PCIT) in the treatment of young children's behavior problems. A randomized controlled study. Plos ONE, 11(9), 1-19. doi:10.1371/journal.pone.0159845

Briggs, H. E., Sharkey, C., \& Briggs, A. C. (2016). The contributions of Applied Behavior Analysis and behavior theory to innovative research and practice cultures in social work. Journal Of Evidence-Informed Social Work, 13(4), 345353. doi:10.1080/23761407.2015.1086710

Coolican, J., Smith, I. M., \& Bryson, S. E. (2010). Brief parent training in pivotal response treatment for preschoolers with autism Jamesie Coolican, Isabel M. Smith, and Susan E. Bryson Parent training in PRT. Journal Of Child Psychology \& Psychiatry, 51(12), 1321-1330. doi:10.1111/j.1469-7610.2010.02326.x

Foxx, R. M. (2008). Applied behavior analysis treatment of autism: The state of the art. Child and adolescent psychiatric clinics of North America, 17(4), 821-834.

Graziano, P., Bagner, D., Slavec, J., Hungerford, G., Kent, K., Babinski, D., \& Pasalich, D. (2015). Feasibility of Intensive Parent-Child Interaction Therapy (I-PCIT): Results from an open trial. Journal Of Psychopathology \& Behavioral Assessment, 37(1), 38-49. doi:10.1007/s10862-014-9435-0

Grindle, C. F., Kovshoff, H., Hastings, R. P., \& Remington, B. (2009). Parents' experiences of home-based applied behavior analysis programs for young children with autism. Journal of Autism and Developmental Disorders, 39(1), 42 56.

Jacobs-Lawson, J. M., \& Hershey, D. A., (2002). Concept maps as an assessment tool in psychology courses. Teaching of Psychology, 29(1), 25-29.

Lord, C., \& McGee, J. P. (Eds.). (2001). Educating children with autism, Committee on Educational Interventions for Children with Autism. Washington, DC: National Academy Press.

Masse, J., McNeil, C., Wagner, S., \& Quetsch, L. (2016). Examining the efficacy of Parent-Child Interaction Therapy with children on the Autism Spectrum. Journal Of Child \& Family Studies, 25(8), 2508-2525. doi:10.1007/s10826-016-0424-7

McDiarmid, M. D., \& Bagner, D. M. (2005). Parent Child Interaction Therapy for children with disruptive behavior and developmental disabilities. Education \& Treatment Of Children, 28(2), 130-141.

Nefdt, N., Koegel, R., Singer, G., \& Gerber, M. (2010). The use of a self-directed learning program to provide introductory training in Pivotal Response Treatment 
to parents of children with Autism. Journal Of Positive Behavior Interventions, 12(1), 23-32.

Smith, T., Donahoe, P. A., \& Davis, B. J. (2000). The UCLA young autism project. In S. J. Handleman \& S. L. Harris (Eds.), Preschool education programs for children with autism (pp. 29-48). Austin, TX: Pro-Ed.

Stahmer, A. (1999). Using pivotal response training to facilitate appropriate play in children with autistic spectrum disorders. Child Language Teaching \& Therapy, 15(1), 29-40. 


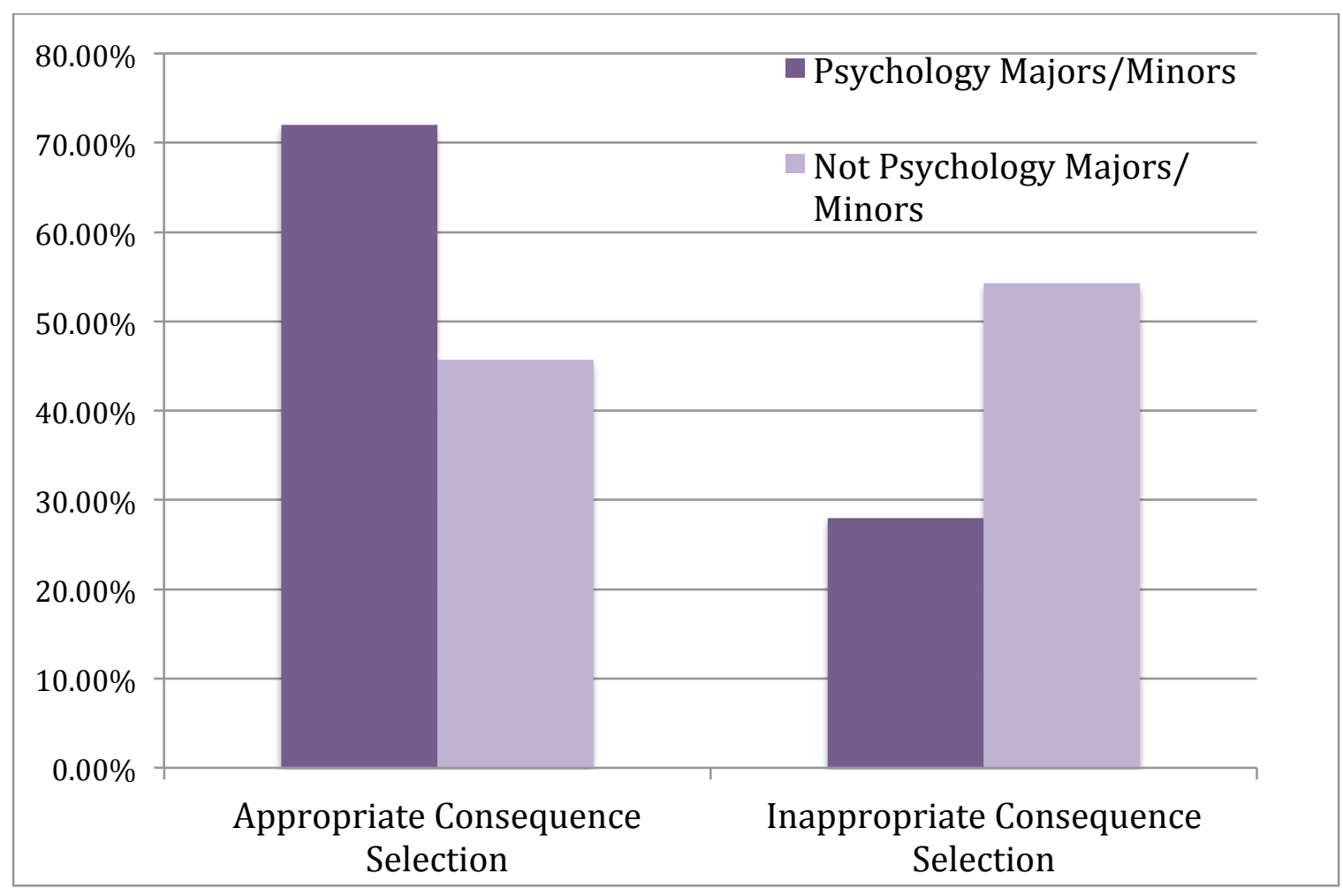

Figure 1. Proportion of students who were Psychology Majors and Minors or not selecting either an appropriate or inappropriate Consequence option. 


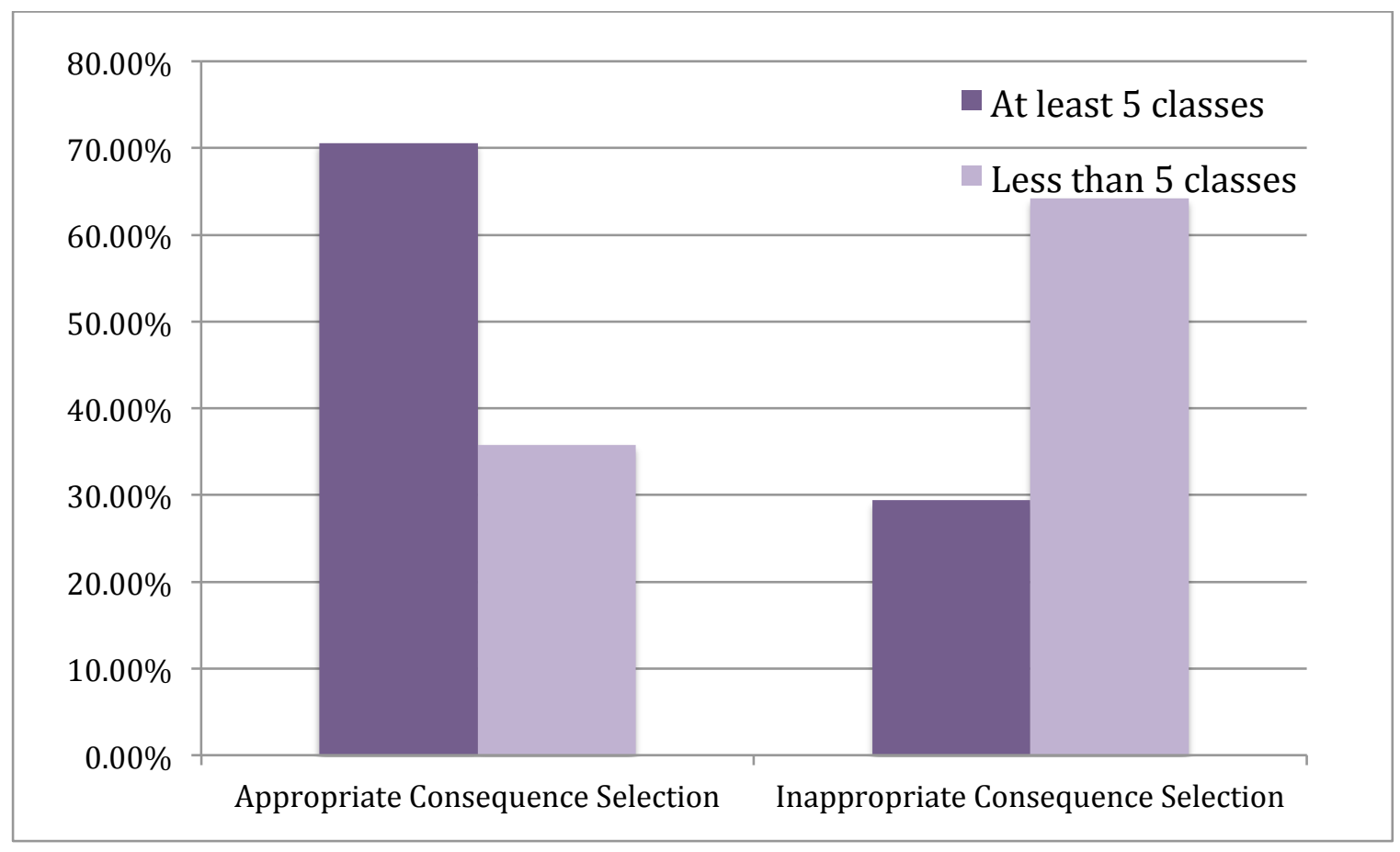

Figure 2. Proportion of students who selected either an appropriate or inappropriate Consequence option based on number of classes taken. 


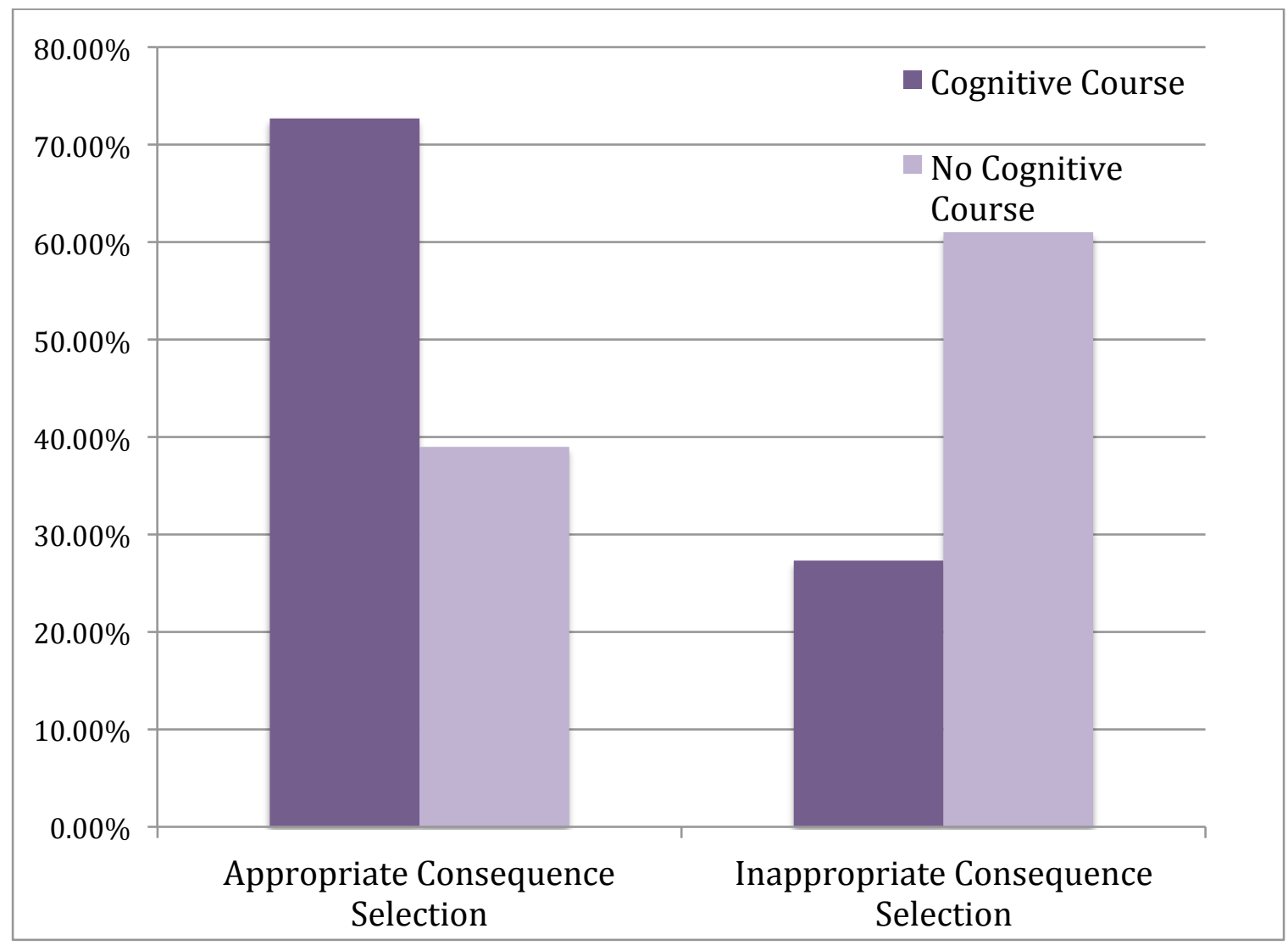

Figure 3. Proportion of students who selected either an appropriate or inappropriate Consequence option based on whether or not they had taken Cognitive Psychology. 


\section{Appendix A}

Survey

Next, you will be asked to read through three scenarios describing the behavior of a child on the Autism Spectrum. Each scenario is laid out as follows:

$A=$ the Antecedent (what precedes or elicits the behavior)

$\mathbf{B}=$ the Behavior (what the child does)

$\mathbf{C}=$ the Consequence (what will happen to the child as a consequence of engaging in the behavior)

In treating Autism and Related Disorders, the consequence implemented is designed to reduce the likelihood that the unwanted target behavior will occur in the future. You will be presented with the Antecedent, Behavior, and potential Consequences. Your task is to rank the provided consequences from $1-5$, where 1 is the most preferred option and 5 is the least preferred option.

\section{Situation 1}

A: "Clean up" after playing with trains for 5 minutes

B: Child stands up, reaches down, grabs plastic train tracks, swings them around and releases them, before running into room and slamming door closed.

C: time out)

Focus on parent involvement: ask parents to implement typical punishment (ex. $\overline{\text { break }}$

Focus on escape behavior: wait for child to calm down, prompt them to ask for a

Focus on completion: have child focus on cleaning up train set

Focus on attention/access-seeking behavior: ignore and begin other activity to entice interest again

Focus on situation: re-set up train tracks quickly and continue to play with child once they've calmed down, and repeat Antecedent instruction to see if behavior will repeat

\section{Situation 2}

A: "All done" and turn off iPad video

B: Child runs to next room, drops to carpeted floor, and screams.

$\mathrm{C}$ : time out)

Focus on parent involvement: ask parents to implement typical punishment (ex. break

Focus on escape behavior: wait for child to calm down, prompt them to ask for a

Focus on completion: allow child to finish tantrum, prompt them to ask for more time on the iPad

Focus on attention/access-seeking behavior: ignore and begin other activity to entice interest again

Focus on situation: re-play video and watch it until child approaches, and then repeat Antecedent instruction and turn off the iPad video to see if behavior will repeat 


\section{Situation 3}

A: "Check your schedule" when it's time to do work

B: Child runs to schedule, tears it off of the wall and throws it onto the ground, while screaming

$\mathrm{C}$ :

Focus on parent involvement: ask parents to implement typical punishment (ex. time out)

Focus on escape behavior: wait for child to calm down and continue with next activity (originally listed on schedule)

Focus on completion: allow child to finish screaming, prompt them to pick up the schedule and complete selection of next item

Focus on attention/access-seeking behavior: ignore and begin other activity to entice interest again

Focus on situation: set up schedule alone and play with reinforcing activity until child has gained interest. Then repeat Antecedent instruction to see if behavior will repeat. 